\title{
Identificación morfológica de Culicoides spp descritos como transmisores de Orbivirus capturados en granjas de ovinos en Pucallpa, Perú
}

\author{
Morphological IDENTIFICATION OF Culicoides Spp DesCribed as Orbivirus \\ transmitters trapped in Sheep farms in Pucallpa, Peru \\ Dennis Navarro M. ${ }^{1}$, Hermelinda Rivera G. ${ }^{1}$, Abraham Cáceres L. ${ }^{2,3}$, \\ Juan Rondón E. ${ }^{4}$
}

\section{RESUMEN}

\begin{abstract}
El objetivo del presente estudio fue identificar ceratopogónidos del género Culicoides (Latreille, 1809) involucrados en la transmisión de Orbivirus. Las capturas se realizaron en dos granjas ubicadas en la localidad de Santa Rosa de Lima, Ucayali, Perú; área que se caracteriza por la presencia de arbustos, árboles, humedales y charcos, así como por la crianza de ovinos de pelo, caballos y aves de corral. Durante tres noches consecutivas se capturaron 7930 ejemplares de Culicoides spp mediante cinco trampas de luz tipo CDC con luz ultravioleta instaladas próximos a los rebaños. La identificación de los Culicoides se hizo con el apoyo de atlas de fotografías del ala de Culicoides neotropicales y claves taxonómicas para cada especie. Cinco especies fueron registradas dentro del subgénero Hoffmania (grupos Guttatus e Hylas) y otro sin clasificación de subgénero (grupo Fluvialis). Se identificaron 7839 (98.8\%) ejemplares de Culicoides hembras y 91 (1.2\%) machos. Según la abundancia relativa, la principal especie fue C. insignis (94.8\%), seguido por C. foxi (3.2\%) y C. ocumarensis (1.3\%). Otras especies como C. pseudodiabolicus, C. hylas y $C$. leopoldoi se presentaron en densidades menores de $0.5 \%$. También se observó un Culicoides $\mathrm{sp}(\mathrm{n}=31)$ que está en proceso de identificación.
\end{abstract}

Palabras clave: ceratopogónidos; chinches; Culicoides; ovinos; trópico; Pucallpa; Perú

${ }^{1}$ Laboratorio de Microbiología y Parasitología Veterinaria, Facultad de Medicina Veterinaria, Universidad Nacional Mayor de San Marcos, Lima, Perú

${ }^{2}$ Departamento Académico de Microbiología Médica e Instituto de Medicina Tropical «Daniel A. Carrión», Facultad de Medicina, Universidad Nacional Mayor de San Marcos, Lima, Perú

${ }^{3}$ Laboratorio de Entomología, Instituto Nacional de Salud, Lima, Perú

${ }^{4}$ Estación Experimental Pucallpa del Centro de Investigaciones IVITA, Universidad Nacional Mayor de San Marcos, Ucayali, Perú

${ }^{5}$ E-mail: dennis.navarro1@unmsm.edu.pe

Recibido: 12 de julio de 2017

Aceptado para publicación: 22 de noviembre de 2017 
The aim of the present study was to identify ceratopogonids of the genus Culicoides (Latreille, 1809) involved in the transmission of Orbivirus. The catches were made in two farms located close to Santa Rosa de Lima village, Ucayali, Peru, an area characterized by the presence of shrubs, trees, and wetlands, as well as the breeding of hair sheep, horses and poultry. During three consecutive nights, 7930 specimens of Culicoides spp were captured through five CDC ultraviolet light traps installed next to the flocks. The identification of the Culicoides was done with the support of atlases of photographs of the wing of Neotropical Culicoides and taxonomic keys for each species. Five species were registered within the subgenus Hoffmania (Guttatus and Hylas groups) and another without subgenus classification (Fluvialis group). It was identified 7839 (98.8\%) female Culicoides and $91(1.2 \%)$ males. According to relative abundance, the main species was C. insignis (94.8\%), followed by C. foxi (3.2\%) and C. ocumarensis (1.3\%). Other species such as $C$. pseudodiabolicus, $C$. hylas and $C$. leopoldoi were present in densities lower than $0.5 \%$. Also, it was observed a Culicoides $\mathrm{sp}(\mathrm{n}=31)$ that is in the process of identification.

Key words: ceratopogonids; biting midges; Culicoides; sheep; tropics; Pucallpa; Peru

\section{INTRODUCCIÓN}

El género Culicoides Latreille 1809 pertenece a la orden Diptera, familia Ceratopogonidae. Comprenden alrededor de 1400 especies clasificadas en 31 subgéneros y 38 grupos sin clasificación (Harrup et al., 2015). Los Culicoides son dípteros de 1 a 5 $\mathrm{mm}$ de longitud, se les localiza en áreas agrícolas, ganaderas y bosques, así como en zonas periurbanas (Purse et al., 2015) y en zonas altitudinales que van desde el nivel del mar hasta los $4200 \mathrm{msnm}$ (Spinelli y Borkent, 2004). Los adultos, tanto machos y hembras, se alimentan del néctar, azúcar y polen de las flores; sin embargo, las hembras son, además, hematófagas, pues necesitan ingerir sangre para madurar y desarrollar sus ovarios y huevos (Mellor et al., 2000). Aproximadamente, el 96\% de las especies de Culicoides se alimentan de sangre de vertebrados, principalmente de mamíferos e incluso de humanos, de allí que desempeñan un rol importante en la transmisión de patógenos virales y parasitarios de interés médico y veterinario (Meiswinkel et al., 2004).
Se han sido aislado más de 50 arbovirus de Culicoides spp de importancia en medicina veterinaria y muchos de ellos pertenecen a las familias Bunyaviridae (20 virus), Reoviridae (19 virus) y Rhabdoviridae (11 virus) (Mellor et al., 2000), de los cuales se destacan el virus (a) de la lengua azul (VLA) considerada como enfermedad de declaración obligatoria a la Organización Mundial de Sanidad Animal (OIE, 2017), (b) de la enfermedad hemorrágica epizoótica (VEHE), (c) de la peste equina africana (PEA), (d) de Oropouche (ORO), (e) de la estomatitis vesicular (VEV), (f) de Schmallenberg (SBV), etc. cuya distribución geográfica se va incrementando debido al cambio climático y calentamiento global, generando la emergencia o reemergencia de estos agentes virales (Hunter, 2003; Maclachlan y Mayo, 2013). Tanto la expansión como el incremento de la densidad poblacional de los vectores han dado lugar al incremento y a la persistencia de los arbovirus durante la estación del invierno, lo que ha permitido ampliar su rango de distribución geográfica (Purse et al., 2005). 
El VLA pertenece al grupo Orbivirus, que son transmitidos por algunas especies de Culicoides. En el Perú ha sido detectado en ovinos aparentemente sanos (Rosadio et al., 1984) y en huanganas de labio blanco (Tayassu pecari) de Madre de Dios (Rivera et al., 2013). Así mismo, en el Perú se han registrado 33 especies de Culicoides, pero su rol como vectores de algún agente viral no ha sido demostrado hasta el momento (Felippe-Bauer et al., 2008). Los trabajos arriba enumerados no mencionan la presencia de $C$. insignis, descrito como vector del VLA y posiblemente de otras especies de Orbivirus en América Latina. Por tanto, el objetivo del presente estudio fue identificar morfológicamente los ejemplares de Culicoides spp capturados en granjas de ovinos de la provincia de Pucallpa (Ucayali, Perú) en un contexto de vigilancia entomológica de importancia en Medicina Veterinaria.

\section{Materiales y Métodos}

Capturas de Culicoides se llevaron a cabo durante tres días consecutivos en noviembre de 2016, época de primavera, en dos granjas donde se crían ovinos de pelo, equinos y aves de corral en la localidad de Santa Rosa de Lima ( $8^{\circ} 3350.7$ LS - 745509.7 LO), distrito de Campo Verde, provincia de Coronel Portillo, departamento de Ucayali, Perú, ubicada a $193 \mathrm{msnm}$. Las granjas se caracterizaban por la presencia de una vivienda rural rodeada de árboles y arbustos y con presencia de charcos y humedales. No se registraron precipitaciones pluviales durante los días de captura.

Las capturas fueron exclusivamente nocturnas mediante cinco trampas de luz (CDC) con luz ultravioleta desde las 17:00 hasta las 05:00 horas del día siguiente. Las trampas se instalaron en las ramas de los árboles, a 1.5 y $2 \mathrm{~m}$ del suelo, y cerca de los lugares de encierro y descanso de los ovinos. Los insectos capturados en cada trampa fue- ron traspasados a frascos de $250 \mathrm{ml}$ conteniendo alcohol $70 \%$ para su conservación y fueron trasladados al Laboratorio de Virología de la Facultad de Medicina Veterinaria de la Universidad Nacional Mayor de San Marcos en Lima, para su identificación morfológica, a través de un estereoscopio y microscopio.

La identificación de los insectos se basó en los siguientes criterios: 1) forma y tipo de ojos, 2) antenas, y 3) tipo, forma y pigmentación de las alas membranosas, según los lineamientos de Wirth et al. (1988), Spinelli (1993); Spinelli et al., (2005), Borkent y Spinelli (2007), Perruolo (2009) y de Sousa Farias et al. (2016).

\section{Resultados}

Se capturaron 7930 ejemplares del género Culicoides, siendo 7839 (98.8\%) hembras y 91 (1.2\%) machos (Cuadro 1). Se identificaron seis especies: Culicoides insignis, C. foxi, C. pseudodiabolicus y $C$. ocumarensis del subgénero Hoffmania Fox, 1948 del grupo Guttatus; una especie del grupo Hylas: Culicoides hylas, y la especie Culicoides leopoldoi $(\mathrm{n}=1)$ del grupo Fluviales sin subgénero establecido (Cuadro 1, Fig.1). Además, una especie que se encuentra en proceso de identificación. La especie más frecuente fue $C$. insignis $(94.8 \%)$ seguido de C. foxi (3.2\%).

\section{Discusión}

La presencia de ovinos, caballos y aves de corral sirven como fuente de alimento para los mosquitos, en particular de las hembras que se encuentran en constante búsqueda de alimento (sangre) para la maduración de sus huevos (Mellor et al., 2000; Kiehl et al., 2009).

Se tiene información que la proporción de hembras y machos Culicoides varía según la ubicación de las trampas (Kirkeby et 
Culicoides del subgenero Hoffmania

Grupo Guttatus

Culicoides insignis Lutz, 1913

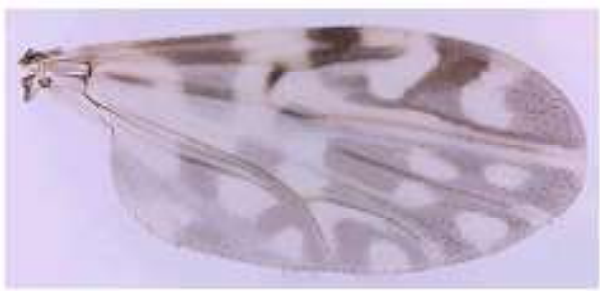

Culicoides pseudodiabolicus Fox, 1956

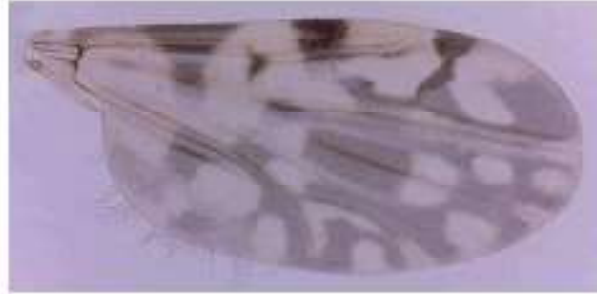

Culicoides foxi Ortiz, 1950

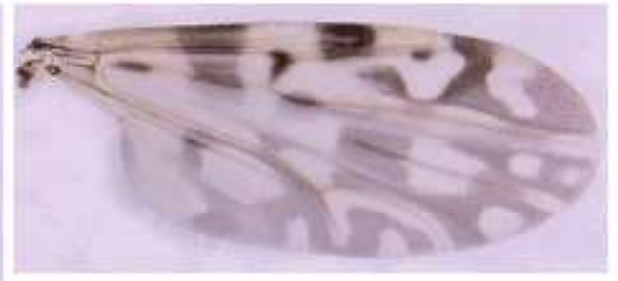

Culicoides ocumarensis Ortiz, 1950

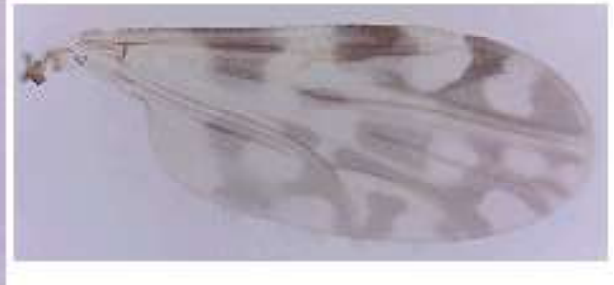

Grupo Hylas

Culicoides hylas Forattini, 1957

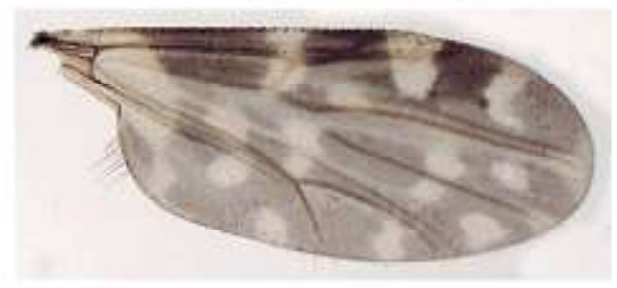

Culicides sin subgénero establecido

Grupo Fluvialis

Culicoides leopoldoi Ortiz, 1951

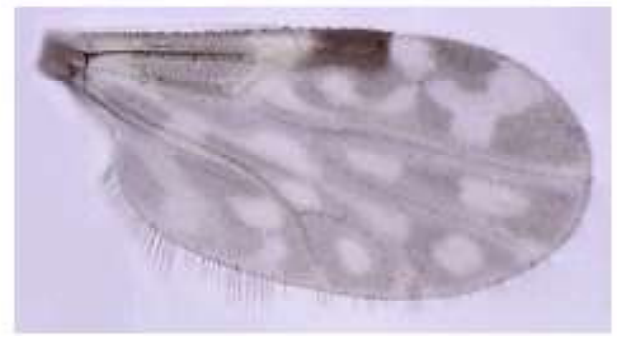

Figura 1. Fotografías de las alas de las especies de Culicoides capturadas en Pucallpa, Ucayali (noviembre, 2016) 
Cuadro 1. Subgénero, grupo y especies de Culicoides capturados de dos granjas de ovinos (Pucallpa, Ucayali, noviembre 2016)

\begin{tabular}{lllccc}
\hline \multirow{2}{*}{ Subgénero } & \multirow{2}{*}{ Grupo } & Especie & Hembras & Machos & Total \\
\cline { 5 - 6 } & & & $(\mathrm{n})$ & $(\mathrm{n})$ & $\mathrm{n}$ \\
\hline Hoffmania & \multirow{2}{*}{ Guttatus } & C. insignis & 7430 & 89 & 7519 \\
& & C. foxi & 251 & 1 & 252 \\
& & C. ocumarensis & 100 & 1 & 101 \\
& & C. pseudodiabolicus & 25 & 0 & 25 \\
& \multirow{2}{*}{ Hylas } & Culicoides sp $^{1}$ & 31 & 0 & 31 \\
Sin ubicación & Cluvialis & C. hylas & 1 & 0 & 1 \\
\hline Total & & & 1 & 0 & 1 \\
\hline
\end{tabular}

${ }^{1}$ Ejemplares de Culicoides en proceso de identificación

al., 2013b). En el presente estudio las trampas fueron ubicadas próximos al grupo de ovinos, lo cual favoreció la captura de hembras hematófagas (Baldet et al., 2008; Baylis et al., 2010; Kirkeby et al., 2013a), permitiendo capturar una población de $98.9 \%$ de hembras; resultados similares a los obtenidos por otros autores (Baylis et al., 2010; GarciaSaenz et al., 2011; Carvalho y Silva, 2013).

Dos de las especies capturadas tienen relación con la actividad ganadera: $C$. insignis y C. foxi (Carvalho y Silva, 2013), condiciones presentes en las granjas de colecta. Otra de las especies capturadas fue $C$. pseudodiabolicus, que es una especie relacionada a la presencia de humanos en la cuenca Amazónica (Borkent, 2015). Asimismo, es importante destacar que la especie más frecuente $(94.8 \%)$ fueron hembras de C. insignis, insectos que se alimentan de la sangre de los ovinos constituyendo un riesgo para la transmisión del VLA y otros orbivirus a la población ovina. C. insignis es considerado como el principal vector del VLA en América del Sur (Lager, 2004) y este vector tiene una amplia presencia en la Amazonía peruana (Felippe-Bauer et al., 2008).

En las últimas décadas, la emergencia global y sin precedentes de agentes virales transmitidos al hombre y a los animales por artrópodos, ha generado un enorme progreso en la entomología y la comprensión de la compleja relación entre los ecosistemas, factores climáticos y los ciclos de vida, dinámica de población, etc., de los Culicoides spp (Takken et al., 2008; Tabachnick, 2010). Estos estudios han permitido identificar las principales especies del género Culicoides, como los pertenecientes a los grupos $C$. obsoletus y C. pulicaris, vectores biológicos del virus de lengua azul en Europa y del virus Schmallenberg, que emergió en el 2011 y afectó a los rumiantes domésticos y silvestres de varios países europeos (Falconi et al., 2011; Kameke et al., 2017). En América del Norte y América Central se han identificado a las especies $C$. sonorensis, $C$. insignis (Maclachlan y Guthrie, 2010) y en el continente africano a las especies $C$. imicola y $C$. bolitinos como los principales vectores del VLA (Coetzee et al., 2012). 
En el Perú se han reportado alrededor de 33 especies de Culicoides, incluyendo $C$. insignis Lutz y $C$. paraensis Goeldi en Iquitos, $C$. lahillei Iches en Amazonas, $C$. pusillus Lutz en Cajamarca e Iquitos y $C$. debilipalpis Lutz en Iquitos, Madre de Dios y Cajamarca (Felippe-Bauer et al., 2008), además de dos especies del grupo Paraensis (C. diversus y $C$. peruvianus) mencionado por Felippe-Bauer et al. (2003), pero su asociación con la transmisión de enfermedades infecciosas aún no ha sido realizada. La correcta identificación de las diferentes especies de Culicoides y en particular de $C$. insignis es de suma importancia, ya que debido al cambio climático podrían ampliar su rango de distribución altitudinal a la zona andina donde se desarrolla la mayor producción ovina del país. El presente estudio es uno de los primeros en iniciar la identificación de artrópodos transmisores de enfermedades emergentes en Medicina Veterinaria como el VLA en el contexto de vigilancia entomológica de los Culicoides.

\section{Conclusión}

En el área de Ucayali, Perú, Culicoides insignis, vector del virus de la lengua azul, fue la especie de mayor frecuencia, seguido de $C$. foxi, $C$. ocumarensis y $C$. pseudodiabolicus.

\section{Literatura Citada}

1. Baldet T, Delécolle J-C, Cêtre-Sossah C, Mathieu B, Meiswinkel R, Gerbier G. 2008. Indoor activity of Culicoides associated with livestock in the bluetongue virus (BTV) affected region of northern France during autumn 2006. Prev Vet Med 87: 84-97. doi: 10.1016/ j.prevetmed.2008.06.014

2. Baylis M, Parkin H, Kreppel K, Carpenter S, Mellor PS, McIntyre KM. 2010. Evaluation of housing as a means to protect cattle from Culicoides biting midges, the vectors of bluetongue virus. Med Vet Entomol 24: 38-45. doi: 10.1111/j.1365-2915.2009.00842.x

3. Borkent A, Spinelli GR. 2007. Neotropical Ceratopogonidae (Diptera: Insecta). Sofia, Bulgaria: Pensoft. 199 p.

4. Borkent A. 2015. World species of biting midges (Diptera: Ceratopogonidae). American Museum of Natural History. [Internet]. Available in: http:// wwx.inhs.illinois.edu/files/8413/4219/ 9566/CeratopogonidaeCatalog.pdf

5. Carvalho LPC, Silva FS. 2014. Seasonal abundance of livestockassociated Culicoides species in northeastern Brazil. Med Vet Entomol 28: 228-231. doi: 10.1111/mve. 12043

6. Coetzee P, Stokstad M, Venter EH, Myrmel M, van Vuuren M. 2012. Bluetongue: a historical and epidemiological perspective with the emphasis on South Africa. Virol J 9: 198. doi: 10.1186/ 1743-422X-9-198

7. de Sousa Farias E, Almeida JF, Pessoa FA. 2016. List of Culicoides biting midges (Diptera: Ceratopogonidae) from the state of Amazonas, Brazil, including new records. Check List 12(6): 1-27. doi: 10.15560/12.6.2002

8. Falconi C, Lopez-Olivera JR, Gortázar C. 2011. BTV infection in wild ruminants with emphasis on reed deer: a review. Vet Microbiol 151: 209-219. doi: 10.1016/j.vetmic.2011.02.011

9. Felippe-Bauer ML, Cáceres $A G$, Santos da Silva $C$, Valderrama-Bazan $W$, Gonzales-Perez A, Costa J. 2008. New records of Culicoides Latreille (Diptera: Ceratopogonidae) from Peruvian Amazonian region. Biota Neotrop 8: $33-$ 38. doi: 10.1590/S1676-06032008000200002

10. Felippe-Bauer ML, Cáceres AG, Silva CS, Valderrama-Bazan $W$, Gonzales-Perez A. 2003. Two new Culicoides of the paraensis species group (Diptera: Ceratopogonidae) from the Amazonian region of Peru. Mem Inst Oswaldo Cruz 98: 1051-1058. doi: 10.1590/S0074-02762003000800014 
11. García-Sáenz A, McCarter P, Baylis M. 2011. The influence of host number on the attraction of biting midges, Culicoides spp, to light traps. Med Vet Entomol 25: 113-115. doi: 10.1111/j.13652915.2010.00904.x

12. Harrup LE, Bellis GA, Balenghien T, Garros C. 2015. Culicoides Latreille (Diptera: Ceratopogonidae) taxonomy: Current challenges and future directions. Infec Genet Evol 30: 249-266. doi: 10.1016/j.meegid.2014.12.018

13. Hunter P. 2003. Climate change and waterborne and vector-borne disease. $\mathrm{J}$ Appl Microbiol 94: 37-46. doi: 10.1046/ j.1365-2672.94.s1.5.x

14. Kameke D, Kampen H, Walther D. 2017. Activity of Culicoides spp (Diptera: Ceratopogonidae) inside and outside of livestock stables in late winter and spring. Parasitol Res 116: 881-889. doi: 10.1007/s00436-016-5361-2

15. Kiehl E, Walldorf V, Klimpel S, AlQuraishy S, Mehlhorn H. 2009. The European vectors of Bluetongue virus: are there species complexes, single species or races in Culicoides obsoletus and $C$. pulicaris detectable by sequencing ITS-1, ITS-2 and 18S-rDNA? Parasitol Res 105: 331-336. doi: 10.1007/ s00436-009-1414-0

16. Kirkeby C, Bodker R, Stockmarr A, Lind P, Heegaard PM. 2013a. Quantifying dispersal of european culicoides (Diptera: Ceratopogonidae) vectors between farms using a novel mark-release-recapture technique. PLoS One 8(4): e61269. doi: 10.1371/ journal.pone.0061269

17. Kirkeby C, Graesboll K, Stockmarr A, Christiansen LE, Bodker R. 2013b. The range of attraction for light traps catching Culicoides biting midges (Diptera: Ceratopogonidae). Parasites Vctors 6: 67- 78. doi: 10.1186/1756-33056-67

18. Lager I. 2004. Bluetongue virus in South America: overview of viruses, vectors, surveillance and unique features. Vet Ital 40: 89-93.
19. MacLachlan N, Guthrie A. 2010. Reemergence of Bluetongue, African horse sicknes and other orbivirus diseases. Vet Res 4: 35. doi: 10.1051/vetres/2010007

20. MacLachlan N, Mayo CE. 2013. Potential strategies for control of bluetongue, a globally emerging, Culicoides-transmitted viral disease of ruminant livestock and wildlife. Antiviral Res 99: 79-90. doi: 10.1016/j.antiviral.2013.04.021

21. Meiswinkel R, Gomulski LM, Delecolle JC, Goffredo M, Gasperi G. 2004. The taxonomy of Culicoides vector complexes - unfinished business. Vet Ital 40: 151-159.

22. Mellor PS, Boorman J, Baylis M. 2000. Culicoides biting midges: their role as arbovirus vectors. Annu Rev Entomol 45: 307-340. doi: 10.1146/ annurev.ento.45.1.307

23. [OIE] Organización Mundial de Sanidad Animal. 2017. Enfermedades, infecciones e infestaciones de la Lista de la OIE en vigor en 2017. [Internet]. Disponible en: http://www.oie.int/es/sanidadanimal-en-el-mundo/oie-listed-diseases2017/

24. Perruolo GJ. 2009. Clave de las especies de Culicoides (Diptera: Ceratopogonidae) asociadas con la ganadería en la región Neotropical. Rev Cient Luz 19: 124-133.

25. Purse BV, Carpenter S, Venter GJ, Bellis G, Mullens BA. 2015. Bionomics of temperate and tropical Culicoides midges: knowledge gaps and consequences for transmission of Culicoides-borne viruses. Annu Rev Entomol 60: 373392. doi: 10.1146/annurev-ento-010814020614

26. Purse BV, Mellor PS, Rogers DJ, Samuel AR, Mertens PP, Baylis M. 2005. Climate change and the recent emergence of bluetongue in Europe. Nat Rev Microbiol 3: 171-181. doi: 10.1038/ nrmicro1090

27. Rivera H, Cárdenas L, Ramírez M, Manchego A, More J, Zúñiga A, Romero M. 2013. Infección por orbivirus 
en huanganas (Tayassu pecari) de Madre de Dios. Rev Inv Vet Perú 24: 544550. doi: 10.15381/rivep.v24i4.2738

28. Rosadio RH, Evermann JF, DeMartini JC. 1984. A preliminary serological survey of viral antibodies in Peruvian sheep. Vet Microbiol 10: 91-96.

29. Spinelli G, Ronderos M, Díaz F, Marino $P$. 2005. The bloodsucking biting midges of Argentina (Diptera: Ceratopogonidae). Mem Inst Oswaldo Cruz 100: 137-150. doi: 10.1590/S0074-02762005000200006

30. Spinelli GR, Borkent A. 2004. New species of Central American Culicoides Latrelli (Diptera: Ceratopogonidae) with synopsis of species from Costa Rica. Proc Entomol Soc Wash 106: 361-395.

31. Spinelli GR, Greiner EC, Wirth WW. 1993. The Neotropical bloodsucking midges of the Culicoides guttatus group of the subgenus Hoffmania (Diptera:
Ceratopogonidae). USA: American Entomological Institute. $91 \mathrm{p}$.

32. Tabachnick WJ. 2010. Challenges in predicting climate and environmental effects on vector-borne disease episystems in a changing world. J Exp Biol 213: 946-954. doi: 10.1242/ jeb. 037534

33. Takken W, Verhulst N, Scholte E-J, Jacobs F, Jongema Y, van Lammeren $R, 2008$. The phenology and population dynamic of Culicoides spp in different ecosystems in the Netherlands. Prev Vet Med 87: 41-54. doi: 10.1016/j.prevetmed.2008.06.015

34. Wirth W, Dyce A, Spinelli G 1988. An atlas of wing photographs with a summary of the numerical characters of the neotropical species of Culicoides (Diptera: Ceratopogonidae). USA: American Entomological Institute. 72 p. 